\title{
The visualization of the use of land on the basis of the dynamics of the pedestrian movement from the interval UAV imaging
}

\author{
Maciej Smaczynski ${ }^{\text {a, }}$, Beata Medynska-Gulij ${ }^{\text {a }}$, Lukasz Halik ${ }^{\text {a }}$ \\ ${ }^{a}$ Affiliation for all authors: Department of Cartography and Geomatics, Adam Mickiewicz University, Poznan, Poland \\ maciej.smaczynski@amu.edu.pl,.bmg@amu.edu.pl,lhalik@amu.edu.pl \\ * Corresponding author
}

Keywords: land use, dynamics of pedestrian movement, UAV, interval imaging, cartographic visualizations

\section{Abstract:}

The identification and visualization of the real land use based on the dynamics of pedestrian movement was the issue discussed in the research. The observation of the pedestrian movement was made on the basis of interval imaging from the low flight level of oblique projection obtained from the single observation station. That led to the occurrence of blind spots, i.e. spots covered by trees and other objects, on imaging made from the drone, which made it difficult or impossible to observe the pedestrian movement on the parts of the research area.

In the research the data on the Land and Building Register were used in order to analyze the cadastral and infrastructural construction of the research area. The photogrammetric record was made during the maximum density of the pedestrian movement on the research area of 7 ha, located on the university campus.

The objective of the research was to create cartographic visualizations depicting the real land use with the employment of different mapping methods, diagrams and other forms of graphic presentation of spatial data. The georeference of the imaging obtained was based on the ground control points and its verification was carried out with the use of independent ground control points. The process allowed one to obtain an orthophotomap of the research area with the precision up to $27 \mathrm{~cm}$ in relation to the coordinates of the ground control points, specified by means of GNSS RTK technology. On the basis of the orthophotomap worked out the location of specific pedestrians was determined with the employment of coordinates. Considering the large scale of the research and its objective, it was necessary to present particular pedestrians, using area spatial objects. The transformation of point objects into area objects was possible thanks to suitable methodology and geomatic transformation.

Furthermore, thanks to the imaging of the 10-second interval and the geomatic research method it was possible to aggregate area objects that represented pedestrians into the land use area. Identified areas of 'wild' land use, on which the pedestrian movement was observed outside the specified communication infrastructure, are particularly noteworthy. Moreover, the aggregation allowed one to solve the problem of blind spots.

As a result of the conducted research, numerical statements concerning the area of land use based on the observation of pedestrian movement were obtained, and the acquired spatial data were presented on cartographic visualizations. 